\title{
Rawbot
}

\section{A digital system for AR fabrication of bamboo structures through the discrete digitization of bamboo}

\author{
Ngai Hang Wu $u^{1}$, Marina Dimopoulou ${ }^{2}$, Han Hsun Hsieh ${ }^{3}$, \\ Christos Chatzakis ${ }^{4}$ \\ 1,2,3,4 Bartlett School of Architecture, UCL, UK \\ 1,2,3,4 \{wucharleswu|dimop.marina|will93611|chatzakis.christos\}@gmail.com
}

This paper presents the developments of a method to assemble bamboo poles through mobile algorithmic instructions, based on material analysis, machine learning procedures and applied augmented reality. The methods were evaluated in a series of tests where the trained system was successfully used to propose structurally sound aggregations, according to the given resources. The results suggest potential benefits for completion of house-scale assemblies by untrained users through automated tools.

Keywords: Machine Learning, Object Recognition, Augmented Reality, Digital Fabrication, Discrete Digitization, Bamboo

\section{1 | INTRODUCTION}

In the rural areas of South East Asia, aboriginals have been using raw materials (bio-based materials) as discrete construction elements for hundreds of years. According to Jon Hartley, due to the emerging economy, there is high demand of fast and efficient housing and infrastructure processes. However, the lack of techniques in building modern concrete structures has as a result a lot of low quality and unsafe houses and infrastructures (Hartley, 2017).

This condition triggered us as a team to further develop a method of extracting information from real materials in physical world into a series of data that can be computed in digital tools. The method discretizes local and raw materials, in this case, bamboo in order to be transformed into a sequence of digital input.

The next step was to implement and enhance this logic into an experience that integrates real-time digital processing of data in the construction phase with raw materials. To accomplish this method, parameters such as accessibility, cost, time, and efficiency were taken into consideration. The concept of applying a real-time computation vision method through a phone that includes a well-trained system to detect specific attributes of the materials by reflecting all the previous examined factors. The training included a set of images that were used as inputs in our system, which later can be used as an identification of each parts of the materials.

\section{2 | BACKGROUND}

In current years, advances in the manufacturing area are related to the automation of processes throughout the production chain. One area of automation is the implementation of machine vision. This 


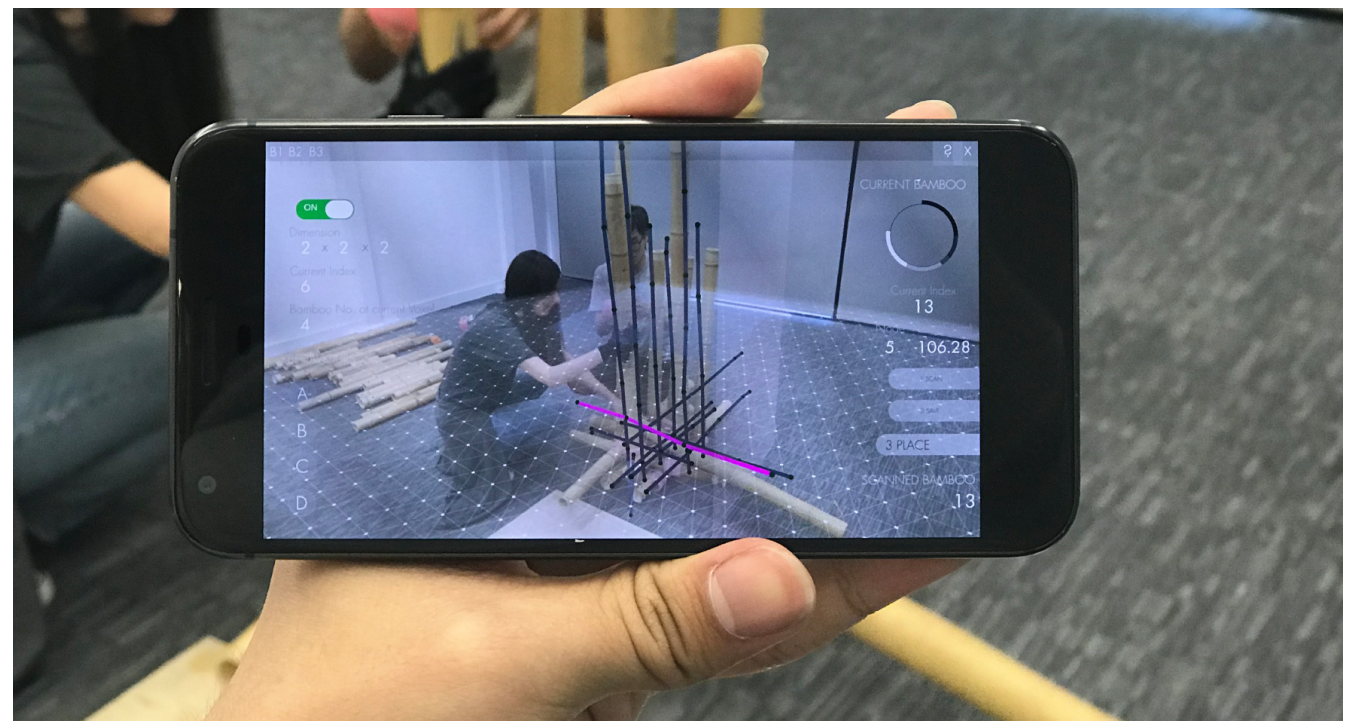

Figure 1

A AR fabrication system of bamboo structure in a smartphone

tool allows the minimization of errors outcoming from human manual operations as well as production costs, the improvement of quality consistency and the increase of productivity. The options for machine vision applications come with some limitations, namely processing power, camera resolution and integration of cost and time. Vincent Conforti from Donnelly Electronics, an American Company founded in 1996 that produced various electronic components, was right when he stated that "Eyes get tired. People make mistakes. I am confident about machine vision systems that have well organized set up". Nowadays, machines and robots are learning to "see". It is a sector of industry that grows because it has high effectiveness, it has became userfriendly and can enhance the productivity of workers through human-machine interaction. Moving towards automation, apart from the traditional industrial methods, new areas of research and technological development are evolving, especially in the architectural space. The dilemma is how people are going to use these tools in order to process the data in a way that is meaningful regarding cost, time and energy and contribute in an innovative way to the contemporary societal processes.

Advanced robotics, artificial intelligence, virtual and augmented reality are some of the breakthrough technologies that represent the fourth industrial revolution which has emerged. These autonomous technological systems are transforming the production processes across industry sectors and propose a cost-efficient alteration of the traditional ways of creation (World Economic Forum, 2017). Therefore, the factor for deciding the location of the production could not be anymore the variations in labour costs. The ongoing digital transformation of the construction industry brings new opportunities for innovative participatory models as well as creates new values at an individual, societal and industrial level, since it fosters multidisciplinary blending (World Economic Forum, 2017). In the case of combining machine learning with augmented reality in architecture, the result would allow the constant analysis of the building performance data and the achievement of greater accu- 
Figure 2

Raw Materials Construction Process

Figure 3 Identification map of safe areas in a city, using machine vision technology (MIT Media Lab, 2015) racy and precision. In addition to this, our research team believes that when the algorithmic platform is trained to recognize and process a respectful amount of data in minimum time, the people involved can focus on more complex tasks.

Our research focuses on the application of machine learning using augmented reality technologies, to assemble bamboo elements. In parallel, it is essential to understand the importance of a study into cybernetics, which lies in certain principles that describe systems in terms of perception and feedback processes. While questioning the role of automation in architecture, it is clear that the answer has its roots in the responsive behaviour of these systems as well as the ever-evolving learning ability of them. The parts of the system are being trained to recognize with high precision the context of images, analyse and convert it into algorithmic instructions for the construction process. Learning machines have made their debut since the middle of the 20th century and have advantages over the human brain such as speed of carrying information, the accessibility to the "forgotten information" and the possibility of interrogating the machine without causing changes in its memory (Rose, 1974).

\section{3 | PRECEDENT STUDIES}

Researchers at MIT Media Lab are using machine learning on street view Google images to teach their system recognizing which areas of the cities are safe and which are not. They feed the computer with visual examples of images from different cities that contain characteristics that define a street as safe. For the accomplishment of this task, people were asked at an online platform to rate the level of safety of the images that they were seeing. The scores were distributed along a scale from 0 to 10 and the computer learnt from shapes, colours, and textures that were presented on the image (MIT Media Lab, 2015). In this way, their system was then able to put a score at an images it have never detected before (MIT Media Lab, 2015). So, the aim is to create a database of perceived safe streets in different cities. This seems to be a powerful tool for city governments, even more in developing countries, as they can measure the condition of the urban environment (MIT Media Lab, 2015). It is obvious that this step contributed in overcoming the limitations regarding the amount of data collected before, to develop similar studies as well as the time spent for the surveys.

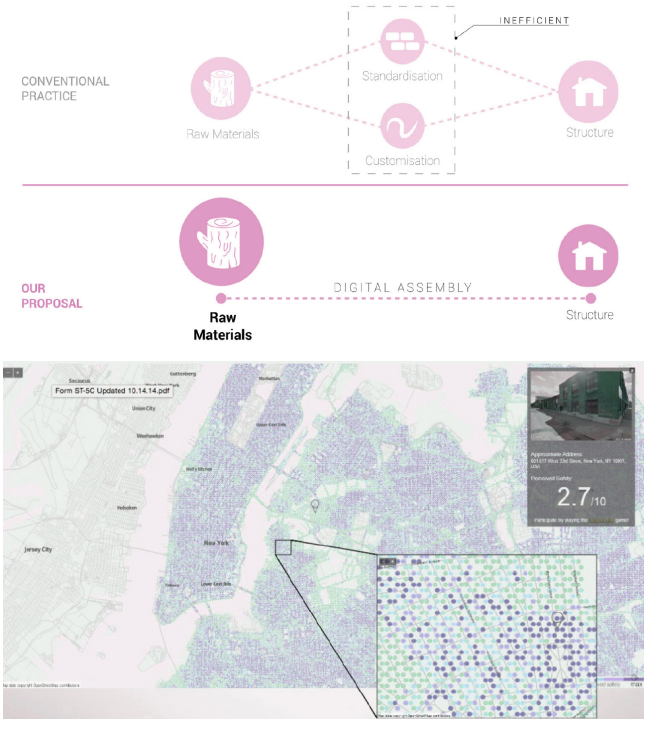

Nowadays, designers have the ability to test an infinite number of models before concluding to the final design that is going to be constructed. When it comes to human preferences, the decisions for satisfying each individual's or at least the largest group of people's aesthetics and comfort levels, take more complex procedures in order to measure (Autodesk, 2018). Swinerton Builders construction company has been using a VR [virtual reality] software that tracks which way people are mostly looking at. Then, having an appropriate amount of data gathered, the system is trained to form specific indications of how furniture could be oriented in space for example, or to put it in an inclusive framework, it is one of the techniques for design optimization (Autodesk, 2018). This 
is a simple concept of supervised learning problem using machine vision technologies in architectural design. Undoubtedly, when the choices increase, the challenges of applications rise as well. The dilemma here is whether there should be a hybrid approach towards automation in design or letting the system be completely decisive, would raise the floor of the design value. An argument on this position is that people are capable of setting the parameters of the problem. Consequently, if the settings of the algorithmic system are intently programmed to produce accurate outputs, the machines can be trusted to calculate, analyze and categorize information.

Our project investigates the implementation of machine vision technologies in the construction process of raw materials. Our case study focuses on bamboo, because it is sustainable and exists in abundance. The starting point was the testing of scanning equipments of different levels of accuracy with bamboo poles. The same process has been followed by other research projects in the past, namely the "Woodchip Barn" project in 2016 at Architectural Association's Hooke Park and a bamboo oriented project that is in progress and is led by professor Lorenzo Rodolfo in the Department of Civil Engineering at UCL. The limitations of this approach were that it was time consuming, the system demanded another object as a reference and usually more than one trials for the same pole were required to complete a full scanning. In addition to this, the equipment and the software were highly priced and an amount of post-scanning process was inevitable in order to extract the information. For this reason, our updated goals included time and cost efficiency, user-friendly device, on-site flexibility and real-time processing. We are creating an app that can be downloaded in smartphone devices and uses machine learning to train a system that can recognize specific attributes of the pole, like the nodes and the ends. Then, based on a structurally optimized computational strategy of aggregating the bamboo poles, the system indicates the position of the pole in the structure. In this way, the automated process contributes to the final- ization of a structurally sound organization of materials based on the availability and the uniqueness of the resources on site.

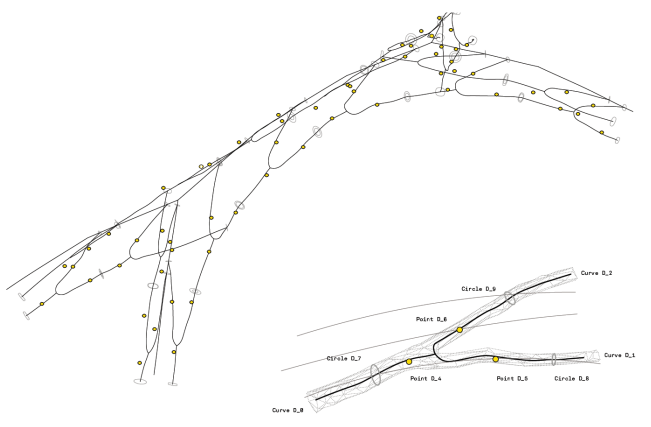

\section{4 | METHODS}

In order to achieve the goal, four key strategies are elaborated: digitizing the pole in order to computationally analyze the irregular shape of natural bamboo poles; studies on fabrication logic to investigate the connection possibilities of different poles; studies on joint system to research about a discrete rigid joint ensuring structure stability; and AR assembly to visualize information to communicate with users and builders.

\subsection{Digitizing the Pole}

Real life construction methods and calculations are heavily dependent on the material. In order to build a brick wall, one must know what type of brick is being used, what are its dimensions and properties and what type of mortar are they using. The same applies in the digital world, were all the calculations are done automatically, however the outcome of these calculations is applied in our physical world. Thus, the actual material properties need to be digitized and fed into the system. In the case that the material is standardized and industrially made its geometry and properties are predetermined ready to be used. When the material is harvested from nature, however, nothing is predetermined, and all the
Figure 4

Fabrication of natural timber in "Woodchip Barn" project at Architecture Association in 2016 
Figure 5

Part of the image library that was used as a data set to train the learning model.

Figure 6

Trained model recognizing random bamboo poles data needs to be extracted. We were able to digitally calculate the structural properties of a bamboo pole as a direct outcome of its geometry which was $3 \mathrm{~d}$ scanned and imported into a script.

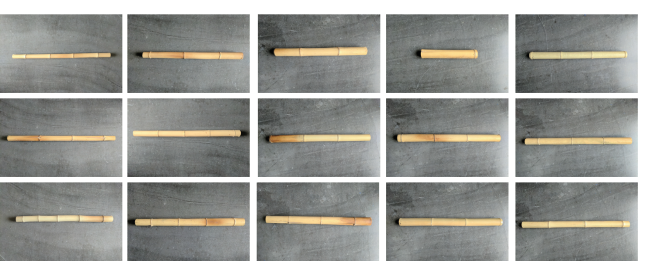

Our project Rawbot investigated the implementation of machine vision technologies in the construction process of raw materials. Our case study focused on bamboo, because it is sustainable and exists in abundance. The starting point was the testing of scanning equipments of different levels of accuracy with bamboo poles. The limitations of this approach were that it was time consuming, the system demanded another object as a reference and usually more than one trials for the same pole were required to complete a full scanning. In addition to this, the equipment and the software were highly priced and an amount of post-scanning process was inevitable in order to extract the information. For this reason, our updated goals included time and cost efficiency, user-friendly device, on-site flexibility and real-time processing.

We are creating an app that can be downloaded in smartphone devices and uses machine learning to train a system that can recognise specific attributes of the pole, like the nodes and the ends. Then, based on a structurally optimised computational strategy of aggregating the bamboo poles, the system indicates the position of the pole in the structure. In this way, the automated process contributes to the finalisation of a structurally sound organisation of materials based on the availability and the uniqueness of the resources on site.

The structure of the bamboo pole has some key points, the nodes, and straight tube-like internode areas, as well as the start and the end points. The proposed system should be able to "see" these key points and recreate a digital representation of the pole which would be accurate enough to produce usable geometry and perform calculations on. For that we used Machine Learning to train an object detector that can, confidently and precisely, detect the end points and the nodes of a bamboo pole. More specifically, we used the Google Tensorflow API and its transfer learning abilities to train a Faster_RCNN_Inception model (Gandhi, 2018). For that we needed a data set, a library of annotated photographs of bamboo. After capturing 230 photographs of poles in varying backgrounds (different indoor and outdoor sets), lighting conditions and configurations (single or multiple poles, overlapping or placed in distance etc.), each image was annotated using the open source labelling software Labellmg (Lin, 2015), carefully placing the tags on our points of interest, being as precise as possible in the size of the bounding box. The next step was the division of the data set into two, the training set and the testing set, in a ratio approximately $80 \%$ and $20 \%$ respectively, resulting to a 182-photograph library training set and a 48-photograph library testing set. After a 4-hour hour training procedure the model was already able to detect the ends and the nodes of every bamboo pole that was presented on camera with an average $98 \%$ accuracy.

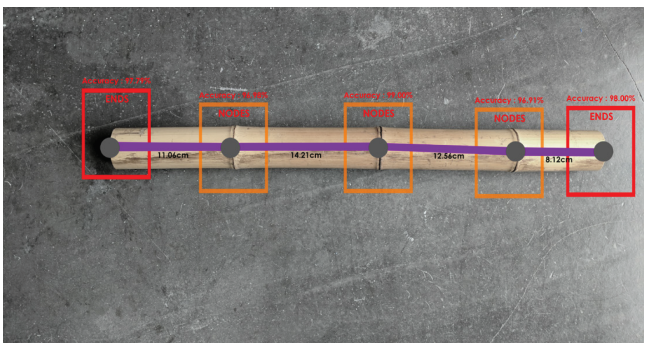

At this point, due to the irregularity and uniqueness of the shape of each bamboo pole, the assumption of a straight core for the algorithmic calculations was made. By connecting the centres of the detections with a straight line and by using the AR capabilities to add the third dimension and internode distances 
the team could recreate a virtual representation of the pole that could be imported in the system.

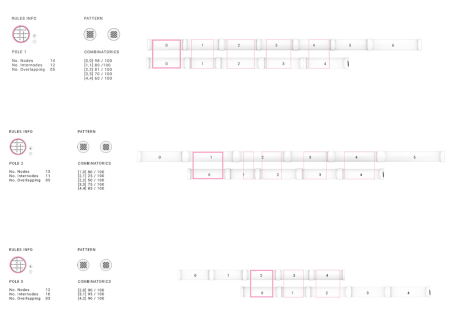

\subsection{Fabrication Logic}

The scope of our approach was the investigation of a computational logic of aggregation of raw materials like bamboo and the management of the irregularity of the shape and the orientation in space, when a mobile phone is the only available equipment. In other words, the team's aim was to create vernacular systems with the minimal infrastructure. Based on a $\mathrm{n}$ initial stress analysis on the necessary volumetric space, we utilised the stress's value and vector's directionality. Each pole placement is defined within voxels. The data that we use in the computational logic come from analysing the information of each single bamboo pole, like the nodes and the internodes areas. The internodes areas were used to put joints and create connecting parts, since they consist the strongest part of the pole, based on the fibres distribution.

The first condition that we set in the algorithm is the calculation of the overlapping percentage of the poles that exist within a voxel till it covers the stress value. We achieved this by comparing each internode area of the pending pole to each internode area of the last pole that was put in structure and get the most optimal result. In order to prevent intersecting poles due to the irregularity of the shapes of bamboos, we get the bounding box of the larger scanned and use its cross section as a unit for subdivision of the voxel. The second condition of the algorithm is the application of a pattern that allows poles coming from different directions to blend and create stronger structures. The sequence of filling the structure is defined based on the most optimal approach regarding time and people. Moreover, we performed real time structural analysis and we place temporarily supports while in the construction process.

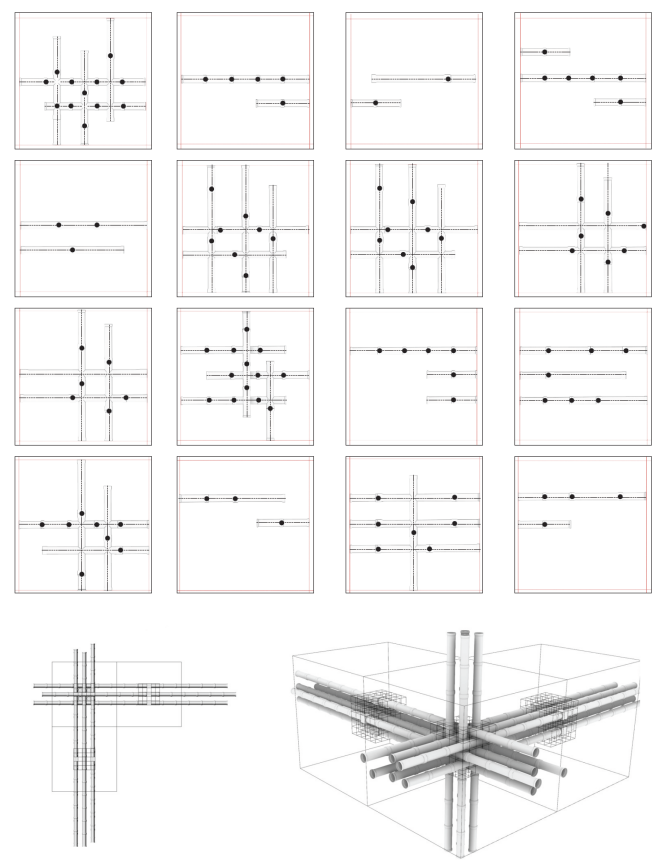

\subsection{Joints}

Untrained workers do not have adequate information on bamboo joinery techniques (Stephane Schroder, 2009). In this case, we had to reinvent the way of connecting bamboo poles. It is described by a jointing system that cannot be deformed and/or lose its original strength.
Figure 7

Connection Logic of different bamboo poles

Figure 8

Studies of fabrication logic of poles and the position of joints

Figure 9 3D prototype testing the possibilities of connections 
Figure 10 Molding model of the joint

Figure 11

Assemble procedure of the joint

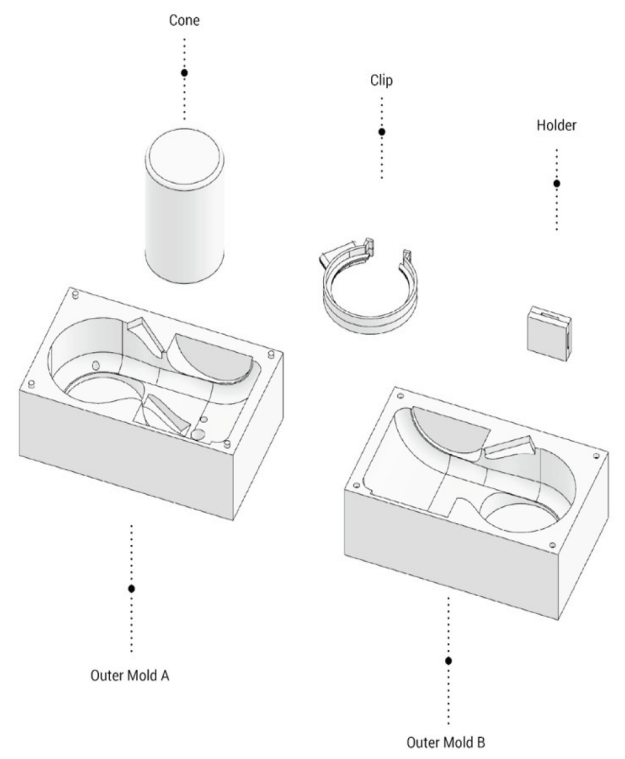

To fabricate a computational product, each bamboo pole had to be firmly connected with each other, in order to create a structurally sound building element. Furthermore, the joint should be designed as a user-friendly and reversible, so that the bamboo could be reused. As the nature of the material had established it prone to cracking, joints that included drilling or screwing were not an option. For this reason, the team developed a custom joint that is strong, durable, adjustable and prevents the pole from damage. It consists of an engraved steel core, two jubilee clips and a rubber envelope. The metallic parts were designed as the structural core of the joint. The team managed to create an adjustable jointing system, combined with jubilee clips, as a hard material and a silicone cover as the envelope, which represent the softer material. The ability of diametrical adjustable jubilee clips gave us the potential to adapt with the different thickness of the bamboo poles. In the meantime, the silicone cover helps to prevent the clip from getting wet, while also preventing the clip from touching the poles directly. The other benefit of the silicone cover is to avoid bamboo from rotating both vertically and horizontally. Additionally, a small metal joint is placed in between to combine two clips together. The outer molds were milled using CNC machine, while the inner parts were $3 \mathrm{D}$ printed. The whole fabrication process takes around 20-35 minutes to dry and be fixed.

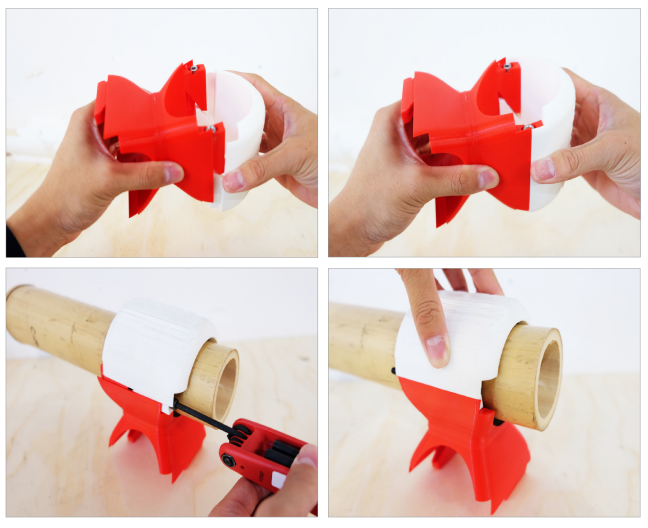

As presented in the diagrams above, this experiment shows how the system adapts to different conditions and behaviors of architectural elements, including vertical and horizontal ways; a corner, a connection, or even a center of a structure.

\subsection{AR Fabrication}

Assuming the scenario in some rural areas in SouthEast Asia because of the size and the cost of the industrial robotic arm, it is very difficult to ship or transport the robots to the site. Therefore, we came up with a construction method using a real-time structural analysis and AR assembly system which is more friendly for human labour assembly. Few onsite workers can work together to construct the whole habitat and structure with the AR instruction from smartphone. In the diagram shown below, we can see that there were two people assembling the structure while another person with a smartphone teaching them how and where the position of the poles should be and how to put the joints. 
Interactive User Designing Interface. First of all, a game-like application is developed for the users to play and move around the space. In this application, the users are able to create their own space by defining the number and position of voxels. At the same time, the system performs real time structural analysis on the voxel space while users are playing. As space grow bigger, the more complex of the structure will be.
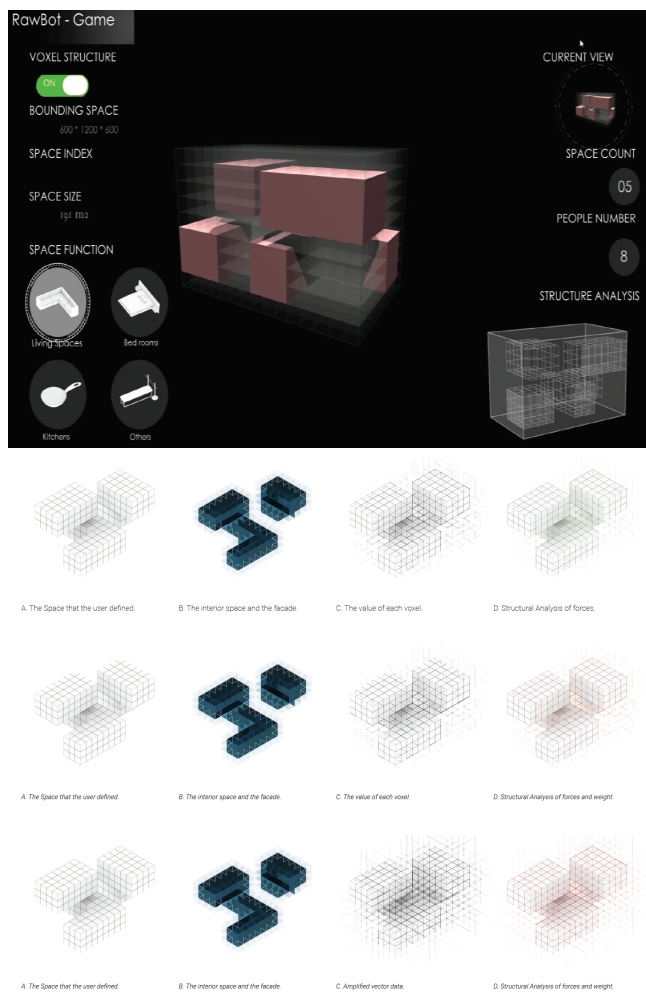

This process helps the system to understand the basic desire of users at the beginning stage. By the inputs from users, the algorithm would read the desired space and run the structural analysis out of that. It visualizes the algorithm behind that generates iterations of housing structure by structural anal- ysis. Interaction between users and computation system encourages involvement of users in early design stage.

Assembly Work Distribute. After establishing the basic housing model based on users' desire, the system starts picking up random bamboo poles and helps fabrication in physical world by providing $A R$ instruction. AR technology is helpful to visualise algorithmic results of the structural analysis behind the system, it teaches users and workers where and how the place the bamboo poles in order to assemble a carefully calculated structure.

Users or workers pick up the first pole onto the scanning area, and the system will automatically recognize the nodes and the ends of the pole by deep learning algorithm as mentioned above. Then the system in the smartphone will tell the builders to place the pole into the right position by projecting signal lines and movement path in the smartphone screen. During this process, a specific worker would be holding the phone in order to give instructions for the builders, in our case, the builders can know the right way to construct the whole structure. In the below images, we can see the process of the construction, in this way, we can construct a complex structure in a very efficient way and only took a very short time. After the construction process, we were able to build a strong column with a few labour and a smartphone.

Column Fabrication. The AR system investigates in a real-time construction process out of raw material which allows convenience and efficiency based on the scenario we had hypothesized above.

The complex structure of a simple column, structurally sounded and can be continue to be assemble, in these images we can see all the connection of each poles, in each poles we can implant more the 3 joints in order to add more poles onto the structure.

\section{5 | CONCLUSION}

The research began to address the bigger issue of emerging economy countries and the culture of construction in the area. Le Corbusier, in Vers Une Ar-
Figure 12

User Application allowing users to define their own space

Figure 13

Real-time structural analysis based on the user-defined voxel space 
Figure 14

Fabrication process with the AR instruction

Figure 15

Column prototype fabricated with AR system chitecture, argued that architects refuse to see the truth that after the industrial revolution, industries like ship making, car making, and air craft making have grown in a dramatically way (Corbusier, 1923). Nowadays, the border between industries and professionals seems to be not as accurate as before, everything is connected with each other in a blurry way. In this project, we were simply roosing a way to bring back traditional raw material construction in an advanced computational way, which requires small infrastructure and small energy cost, but the system itself can adapt to the environment and the local materials. We are proposing that architecture should not be a Top-Bottom process, but simply be architecture without architects.

\section{6 | FUTURE WORKS}

According to the brief introduction by Andrew Witt (2017), the beginning of a period of heightened visibility for an intensive interest in the theoretical possibilities of computation and behavioral networks, which crystallized into specific technologies (Witt, 2017). During this period, architects and designers showed great enthusiasm towards new design method such as cybernetic and network process. As Witt mentioned, the new method such as scripting and programming must be applied into the field of architecture (Witt, 2017). The research began with a bigger issue of emerging economy countries and the culture of raw material constructions using local building elements from the very region. For the next step of the project, an implementation of artificialintelligence functions, including machine learning as a self-awareness for maintenance will be further developed. Before the team is able to apply the system to other material, more technical information will be added to the system, the first thing will be the way which the system recognize the materials, in this design project, the example are bamboos, which this material contains a very obvious structural distribution on the poles, the nodes. If the team wish to develop the system to adapt another type of raw material, such as timber woods or rocks, a different type
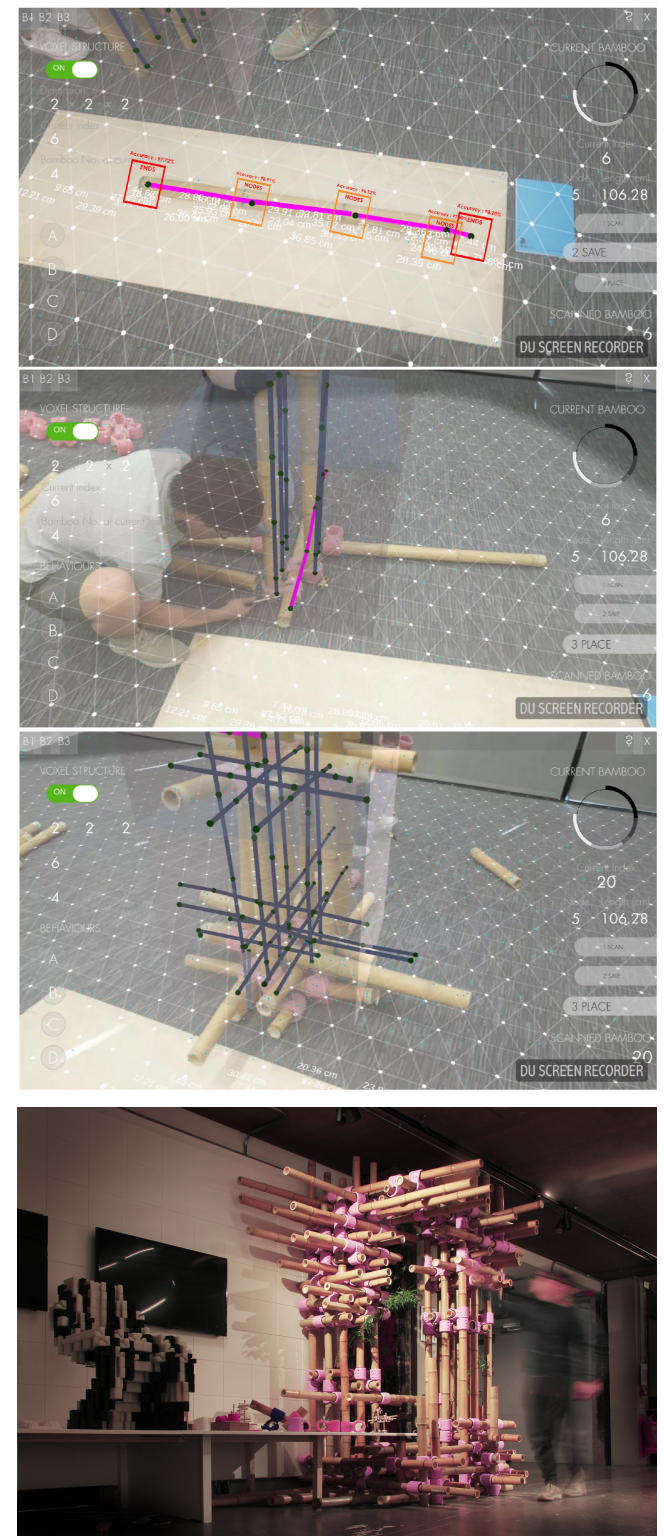
of machine learning workflow needs to be implant as an data base for switching the method of recognizing the structural point distribution of different type of materials. This system will be further developed as our goal is to make the system adaptive to varies of raw materials.

This architectural example is one of our larger version of architectural scale experimentation, to test the system, joints and structural analysis. After several attempt of experiments, we were able to come up with a fully functioning construction system which includes a computer vision via a phone, machine learning application, structural analysis and AR assisted fabrication in the making process.

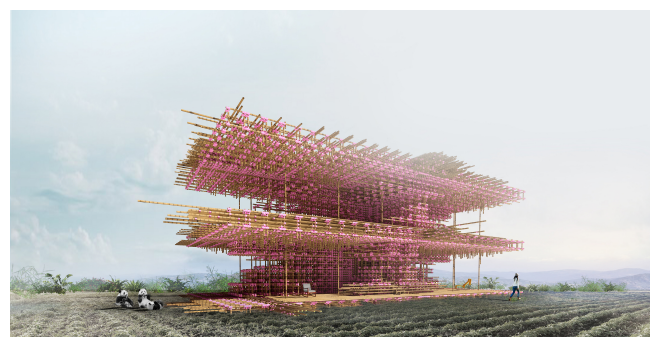

\section{7 | ACKNOWLEDGEMENT}

Our thanks to Gilles Retsin, Manuel Jimenez Garcia, Vicente Soler and Mollie Claypool from Bartlett School of Architecture for their pertinent and insightful advice. Also, our gratitude to Dr. Rodolfo Lorenzo and Dr. Leonel Mimendi from Civil Environmental and Geomatic Engineering Department in UCL for their expert knowledge on bamboo construction properties. Last but not least, we thank Microsoft Azure for providing cloud training platform for our prototypes.

\section{REFERENCES}

Burnett, R 2004, How Images Think, MIT Press

Corbusier, L 1923, Vers une Architecture, Editions Flammarion, Paris, France

Gandhi, R 2018, 'R-CNN, Fast R-CNN, Faster R-CNN, YOLO

- Object Detection Algorithms', Towards Data Sci- ence, 1, p. 1

Hartley, J 2017, 'The Emerging Markets Housing Bubble', Huffpost, 1, p. 1

Lorenzo, R 2017 'BIM Bamboo: a digital design framework for bamboo culms', ICE Publishing, London

van der Lugt, P 2017, Booming Bamboo, Publisher Materia, London

Muklashy, W 2018, 'How Machine Learning in Architecture is Liberating the Role of the Designer', Autodesk, 1, p. 1

Naik, N 2015, How to use computer vision to improve cities, Ph.D. Thesis, MIT Media Lab

Rose, J 1974, The Cybernetic Revolution, Paul Elek (Scientific Books) Ltd.

Rudofsky, B 1987, Architecture without Architects: A Short Introduction to Non-Pedigreed Architecture, University of New Mexico Press

Schröder, S 2009, 'Bamboo Joints and Joinery Techniques', Guadua Bamboo, 1, p. 1

Witt, A 2017, The Machinic Animal, When is the Digital in Architecture, Sternberg Press

[1] http://www3.weforum.org/docs/WEF_Impact_of_th e_Fourth_Industrial_Revolution_on_Supply_Chains_.p df

[2] http://ibuku.com/sharma-springs-residence/

[3] https://www.britannica.com/science/cybernetics

[4] https://github.com/tzutalin/labellmg
Figure 16 Architectural visualization by computational bamboo fabrication 